\title{
A modified BESS model as the effective description of strong electroweak symmetry breaking
}

\author{
Mikuláš Gintner $^{a b c}$, Josef Jurán̆ ${ }^{* c}$ and Ivan Melo ${ }^{a}$ \\ ${ }^{a}$ Physics Department, University of Žilina, Žilina, Slovakia \\ ${ }^{b}$ Science and Research Institute, Matej Bel University, Banská Bystrica, Slovakia \\ ${ }^{c}$ Inst. of Experimental and Applied Physics, Czech Technical University, Prague, Czech Republic \\ E-mail: gintner@fyzika.uniza.sk, josef.juran@utef.cvut.cz, \\ melo@fyzika.uniza.sk
}

\begin{abstract}
Facing the plethora of alternative hypotheses for the mechanism of electroweak symmetry breaking (ESB) it is highly desirable to typify its phenomenology using the effective Lagrangians. The BESS (Breaking Electroweak Symmetry Strongly) model is one of them. The model couples the vector resonances universally to all Standard model (SM) fermion generations. We have modified the model by allowing direct interactions of the vector triplet with the third quark generation only. This is motivated by the extraordinary mass of the top quark which is close to the ESB scale. In addition, we have introduced new Lagrangian terms admitted by the model's symmetries. Our modifications of the BESS model can significantly relax the low-energy limits on the original BESS model's parameters. Further, due to the modifications the new vector resonances became narrower. Here, we present the new model's basic phenomenology and compare it to the original BESS model.
\end{abstract}

The 2009 Europhysics Conference on High Energy Physics,

July 16 - 222009

Krakow, Poland

\footnotetext{
* Speaker.
} 
One essential component of the SM remains a puzzle: the actual mechanism behind the ESB. The Higgs scalar field of a non-zero vacuum expectation value serves as a benchmark hypothesis for the mechanism. Alternative extensions of the SM range from supersymmetric theories with multiple elementary Higgs bosons in their spectra to the theories of new strong interactions which might form bound states of new elementary particles. In addition, a dual-description relation of the extra-dimensional theories to the models of dynamical ESB has entered the scene (see [1] and references therein).

The BESS model [2] effectively describes a Higgsless ESB mechanism accompanied by a hypothetical strong triplet of vector resonances $V_{\mu}^{a}$. The effective description introduces two new free parameters: $g^{\prime \prime}$, which is the $S U(2)_{V}$ gauge coupling, and $\alpha$. The masses of the neutral and charged resonances depend on the parameters, see Fig. 1. Therein we consider the fixed values of
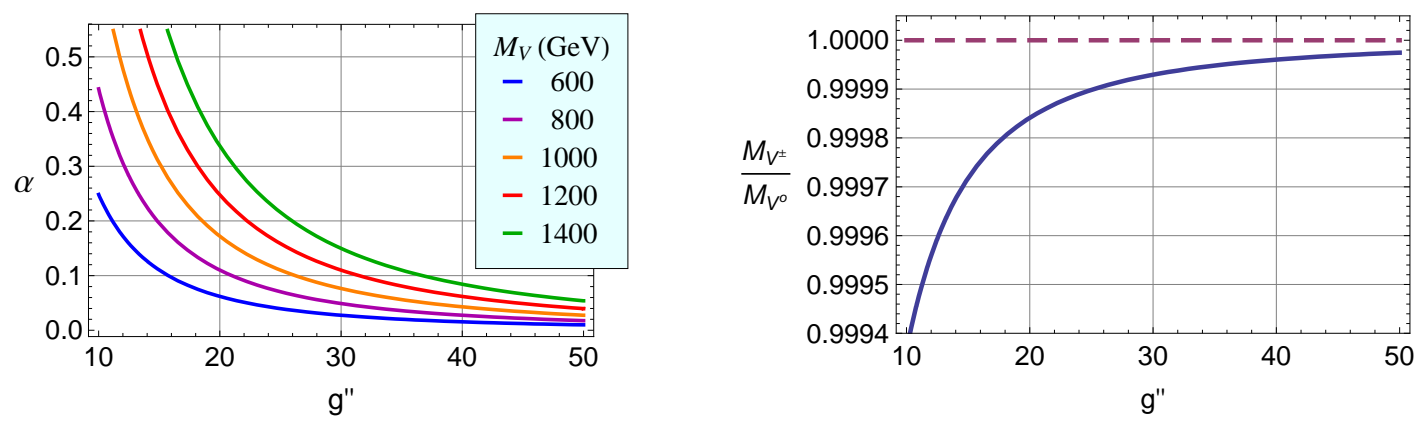

Figure 1: On the left: The relations between $\alpha$ and the $S U(2)_{V}$ gauge coupling $g^{\prime \prime}$ at various fixed masses of $V$. On the right: The splitting of the $M_{V^{ \pm}}$-to- $M_{V^{0}}$ degeneracy as a function of $g^{\prime \prime}$.

the $Z$ and $W$ masses as taken from experiment. To compensate for the non-SM corrections to $M_{Z}$ and $M_{W}$ the parameters $v, g$, and $g^{\prime}$ vary slightly with $g^{\prime \prime}$.

Beside the indirect interactions of the vector resonances to the SM fermions induced by the mixing of $V$ with the SM gauge bosons, the BESS model [2] couples $V$ directly and universally to all SM fermion generations of a given chirality. The direct interactions are parameterized by two free parameters, $b$ for the left fermions, and $b^{\prime}$ for the right fermions. The low energy measurements imply the following limits: $b \lesssim 0.01, b^{\prime} \approx 0$.

We have modified the BESS model [2] by allowing direct interactions of $V$ with the third quark generation only [3]. This is motivated by the extraordinary mass of the top quark which is close to the ESB scale. Thus in our model there are no direct interactions of $V$ to either leptons or to $u, d, c, s$ quarks. The direct interaction of $V$ to the left $(t, b)$ doublet is parameterized by the coupling $b_{1}$ while the direct interaction to $t_{R}$ is parameterized by $b_{2}$. The direct interaction to $b_{R}$ is further modified by a tuning parameter $p, 0 \leq p \leq 1$. In addition, we have introduced new Lagrangian terms admitted by the model's symmetries, thus introducing two additional parameters $\lambda_{1}$ and $\lambda_{2}$ for left and right $(t, b)$ doublets, respectively. The low energy experiment limits read: $g^{\prime \prime} \gtrsim 20$, $-0.003<b_{1}-\lambda_{1}<0.01$, and either $\left|b_{2}-\lambda_{2}\right|<0.008$ assuming $p=1$ or $-0.03<b_{2}-\lambda_{2}<0.04$ if $p=0$. While $\lambda$ terms help to relax the low-energy limits on the parameters of the model we have found that their influence on observables at $E \sim M_{V}$ is negligible.

The vector resonances decay dominantly to the SM gauge bosons and/or to the third generation of quarks. In Fig. 2 we show the total decay widths of $V$. The plots of the partial decay widths 

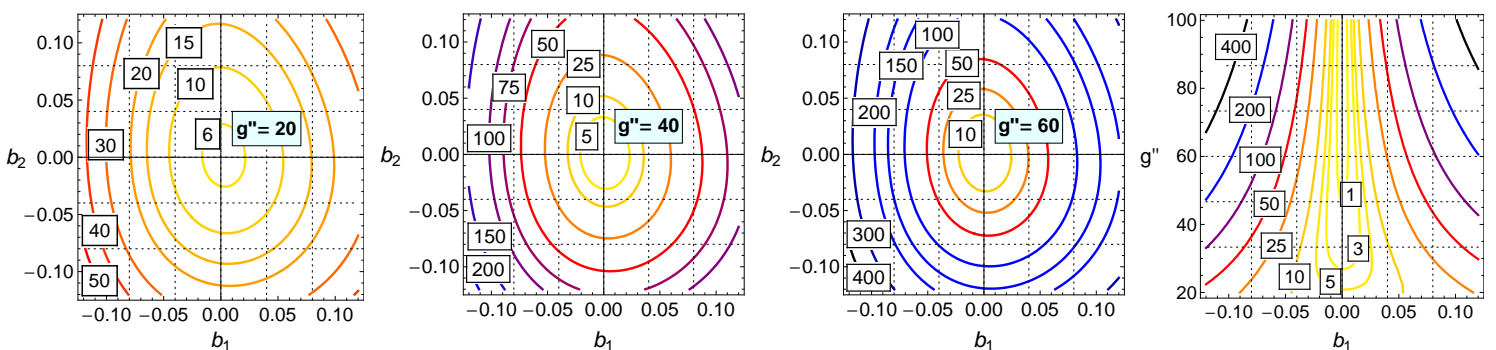

$b_{1}$

$b_{1}$

Figure 2: The our model total decay width contours of $V^{0}$ in the $\left(b_{1}, b_{2}\right)$ parametric space at various values of $g^{\prime \prime}$, and of $V^{ \pm}$in the $\left(b_{1}, g^{\prime \prime}\right)$ parametric space. The contour labels indicate the widths in GeV.
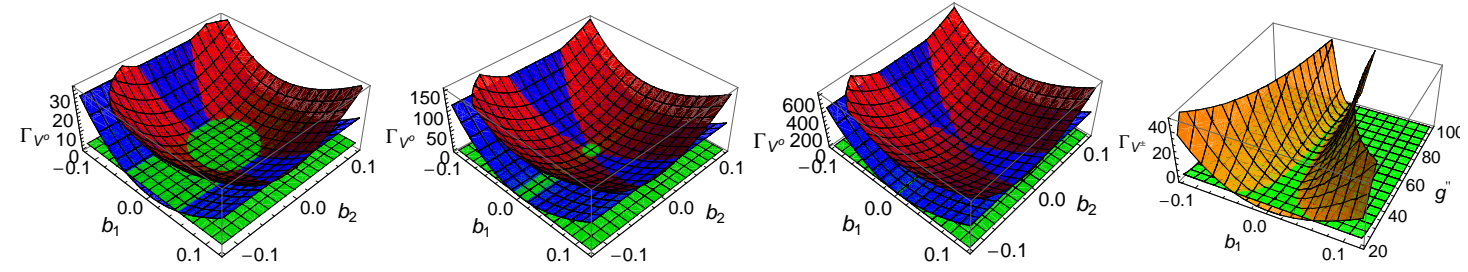

Figure 3: The our model partial decay widths of $V^{0}$ as functions of $b_{1}$ and $b_{2}$ at $g^{\prime \prime}=25,50,100$, from the left to the right, respectively, and of $V^{+}$as functions of $b_{1}$ and $g^{\prime \prime}$. For $V^{0}$, the green, blue, red surfaces correspond to the $W^{+} W^{-}, b \bar{b}, t \bar{t}$ channels, respectively. For $V^{+}$, the green, orange surfaces correspond to the $W^{+} Z, t \bar{b}$ channels, respectively. The partial decay widths are in $\mathrm{GeV}$.
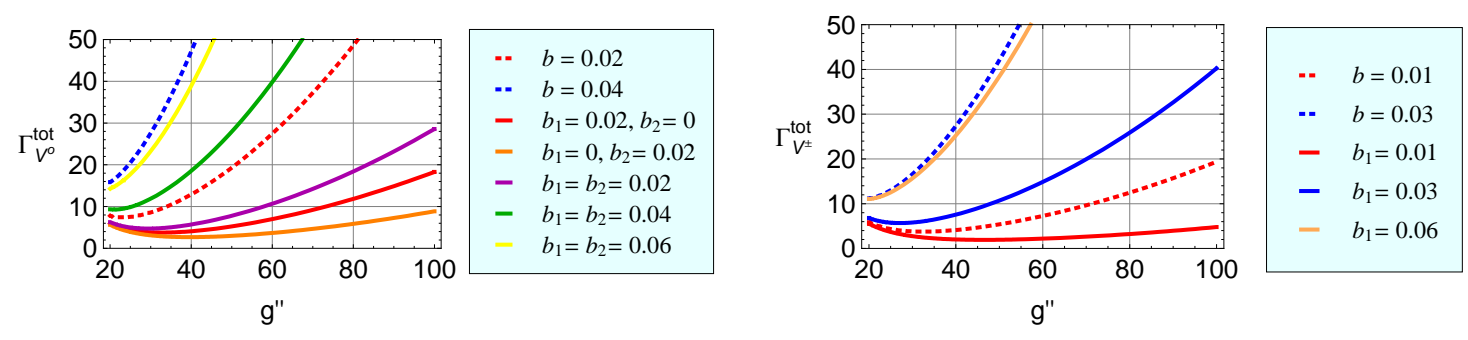

Figure 4: Differences between the original (dotted) and modified (solid) BESS models demonstrated through the total decay widths of $V^{0}$ and $V^{ \pm}$(in $\mathrm{GeV}$ ). The modifications have made the resonances slimmer.

are shown in Fig. 3. Finally, we compare the original and modified BESS models through the total decay widths of $V^{0}$ and $V^{ \pm}$in Fig. 4. In Figs. $2-4$ we have assumed $M_{V^{0}}=1 \mathrm{TeV}$ and $p=0$.

Our preliminary calculations not shown here suggest the potential of some of the LHC processes to detect the new vector resonances. However, a more realistic analysis is needed.

\section{References}

[1] H-C Cheng, in proceedings of 15th Int. Conference on Supersymmetry and the Unification of Fundam. Interactions (SUSY07), Karlsruhe, Germany, 26 Jul - 1 Aug 2007, [hep-ph/ 0710.3407 ].

[2] R. Casalbuoni, S. De Curtis, D. Dominici, R. Gatto, Phys. Lett. B155 (1985) 95; R. Casalbuoni,

S. De Curtis, D. Dominici, R. Gatto, Nucl. Phys. B282 (1987) 235; R. Casalbuoni, P. Chiappetta, S. De Curtis, F. Feruglio, R. Gatto, B. Mele, J. Terron, Phys. Lett. B249 (1990) 130.

[3] M. Gintner, I. Melo, B. Trpišová, Acta Phys. Slov. 56 (2006), 473-483. 